\title{
DISCIPLINARY IMPLICATIONS OF A SYSTEM ARCHITECTING APPROACH TO COLLABORATIVE AIRCRAFT DESIGN
}

\author{
JAN-N. WALTHER, PIER D. CIAMPA AND BJÖRN NAGEL \\ DLR Institute for System Architectures in Aeronautics \\ Hein-Saß-Weg 22, 21129 Hamburg \\ e-mail: \{jan-niclas.walther,pier.ciampa,bjoern.nagel $\} @$ dlr.de
}

Key words: System Architecting, Collaborative Engineering, MDO, CPACS, Computational Aeroelasticity, Preliminary Aircraft Design, Structural Analysis and Design

\begin{abstract}
In the face of growing public awareness of environmental issues such as climate change, the pressure to provide efficient and ecological new air transport solutions is higher than ever on the aviation community. To this aim, unconventional aircraft configurations, which are radically different from the established tube-and-wing architecture, may hold a lot of potential [1]. However, OEMs today usually shy away from such configurations due to the significantly increased uncertainty and entrepreneurial risk connected to such drastic design changes. In order to reduce the risk and increase knowledge about a new configuration, the application of physics-based analyses on a virtual aircraft can add significant value, when applied in the early stages of the design process by bringing new technologies to higher TRLs quickly. Due to the highly multidisciplinary nature of the aircraft design task, the success of this approach largely depends not only on the well-organized handling of the available product data at any point in the design process but also the smart sequencing of the disciplinary contributions based on their mutual dependencies. In this paper, a methodology for an integrated and collaborative approach to preliminary aircraft design is presented. It applies several well established components, such as CPACS (Common Parametric Aircraft Configuration Schema) [2] as a central product data schema and RCE (Remote Component Environment) [3] which enables an automated collaborative approach to aircraft design and combines them with methods from system architecting and model-based engineering [4]. Furthermore, the requirements for a disciplinary analysis and design tool to contribute to an integrated multidisciplinary design process are highlighted. Three examples are given, assuming the perspective of a structural designer:
\end{abstract}

- An unmanned aerial vehicle from the AGILE project [4], where a cross-organizational workflow has been set up in order to perform aero-structural MDO on the wing planform [5].

- A Prandtl-plane configuration from the Parsifal project [6]. Here, a tail plane design and sizing is performed using data collected from a variety of partners.

- A conventional configuration from the InDiCaD project, where the structural layout is designed in tandem with the cabin $[7,8]$.

The example cases demonstrate the initial investment necessary in order to integrate a disciplinary tool into a multidisciplinary environment as well as the potential benefits of being able to perform the analysis within a larger context. 


\section{INTRODUCTION}

For several decades, aerospace research in both industry and academia has been driven by a need to improve efficiency and reduce fuel consumption and emissions. New technologies and disruptive configurations are continuously being proposed, promising lower aircraft weight or improved aerodynamic efficiency. That said, OEMs tend to stick to proven tube-and-wing configurations for new developments or eschew completely new designs altogether, instead exploiting family concepts as much as possible. Given the financial investment required for an aircraft program, this incremental approach is entirely reasonable, as it provides a means to control risk.

However, as the need for green mobility increases, ways must be found to reduce the barriers for bringing innovative design concepts to higher TRLs, making them more attractive for industrial implementation. A promising way forward is to front load the design process. This means, that more diverse and more detailed knowledge of the design is generated during the early design stages, driven primarily through physics-based numerical simulation on virtual aircraft models. As more disciplines besides aerodynamics and structural simulation, such as manufacturing or certification, are being included, the need for simulation tools and disciplinary expertise increases. Additionally, interdisciplinary effects must be taken into account, which means that dependencies between disciplines must be identified and suitable interfaces provided. This is a central aspect of system architecting in collaborative aircraft design.

By combining disciplinary contributions in a smart way and asserting smooth and unambiguous data exchange, even among different organizational units, sites or institutions, interdisciplinary design cycles can be accelerated. This means that, given a fixed time frame, a multidisciplinary design system can be used to explore a larger da

design. Ultimately, this rest

for industrial companies

The German Aerospace

$10,11]$, aiming to establis

from preliminary desig level all the way to certification
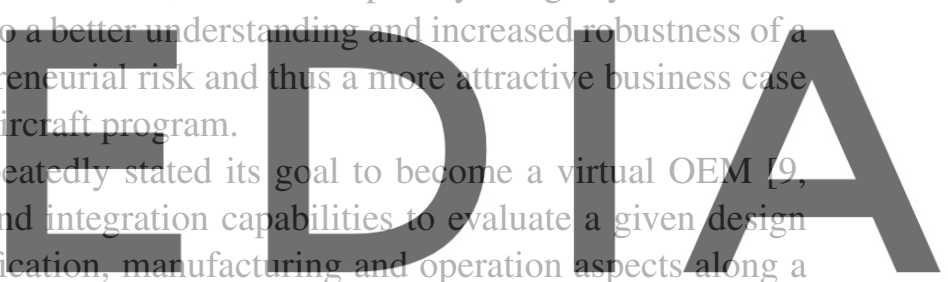

continuous digital thread. Establishing links between disciplinary experts and fostering collaboration are

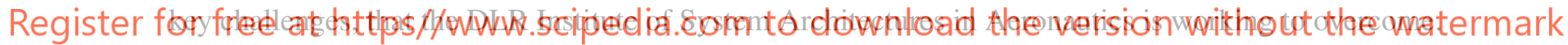

\section{FUNDAMENTALS OF SYSTEM ARCHITECTURE-DRIVEN COLLABORATIVE}

\section{AIRCRAFT DESIGN AT DLR}

The vision to perform collaborative aircraft design has been pursued, at the DLR, since the TIVA (Technology Integration for the Virtual Aircraft) project, which was launched in 2005 [12, 13]. Given its organizational structure, where disciplinary institutes are spatially distributed across Germany, the benefit of sharing analysis services and results remotely is obvious. The technologies initiated during the TIVA project have since been further developed and applied in a string of subsequent projects $[14,15$, $16,17,18,4]$, many of which involving partners from academia and industry outside the DLR. Looking at the integration activities in these projects, four main pillars can be identified, which all of them have in common: A common language for exchanging data, a process integration framework, system architecting capabilities and disciplinary analysis tools along with experts who know how to use them and interpret the results. These will be detailed in the following this section. 


\subsection{CPACS: A Common Language}

The metaphor of a common language for describing the Common Parametric Aircraft Configuration Schema (CPACS) has been strained beyond measure, ever since the publication of the seminal paper by Nagel et al. [19]. It is also remarkably appropriate. In more technical terms, one might rather refer to CPACS as a central data model, but ultimately, like a language, it provides the means for communicating aircraft product information in a way that is at once clear and unambiguous. In cooperation with disciplinary specialists, the format has been designed and refined to serve a wide variety of domains related to preliminary aircraft design, including classical disciplines such as aerodynamics and structures, but also mission simulation or climate impact assessment [2]. Different scales such as fleet aspects can also be considered and the fidelity of the model can be increased by augmenting more specific data as it becomes available.

From an information technology perspective, CPACS builds upon the eXtensible Markup Language (XML), but as indicated by terms like schema and data model, it does not contain data. Instead, a hierarchical structure is provided as an XML schema definition file, in which to store aircraft data in an XML instance of CPACS. The outline of an XML node tree for an example CPACS file is given in figure 1a. The schema is openly available [20], and can be used to validate CPACS XML data sets, i.e. XML files built following the CPACS hierarchy. An important advantage of XML is, that it is easily readable to both humans and machines, which makes it suitable for data exchange between people, as well as software tools.
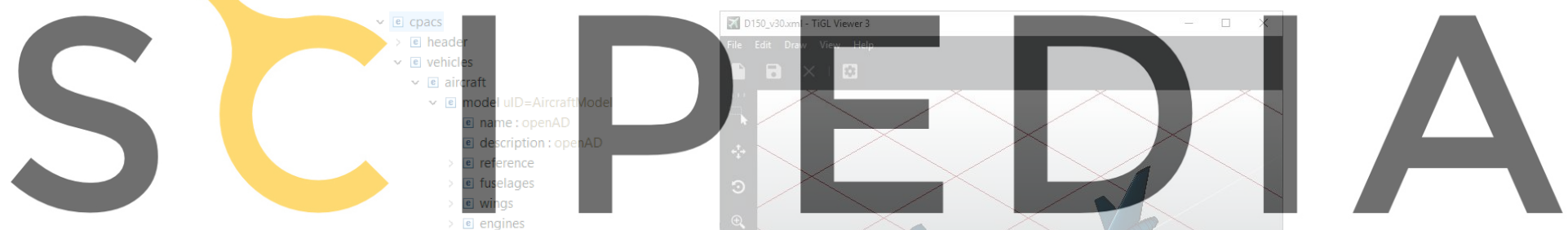

Register for free at https//wwww.scipedia.com to download the version without the watermark

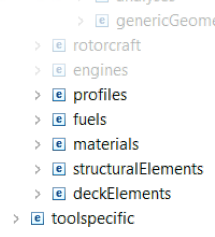

(a) Outline of a CPACS tree

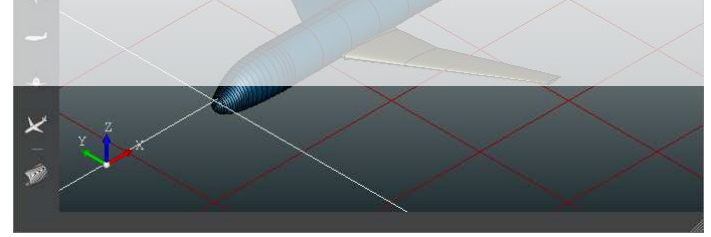

(b) CPACS visualization in TiGL Viewer

Figure 1: CPACS tree structure and visualization

Besides the common form, a key feature of a common language is a consistent interpretation. For aircraft geometry, the de facto standard interpretation is provided by the TiGL (TiGL Geometry Library) library [21]. Leveraging the open source Open CASCADE Technology CAD kernel [22], the ever expanding feature set of TiGL no longer just includes lofting of the wings and fuselage surfaces, but also modeling of primary structure, movables, engine nacelles and more. For many, TiGL may be their first point of contact with CPACS, as the developers also provide the TiGL Viewer, which can be used to 
easily visualize the geometry from CPACS files.

In addition to TiGL, an exhaustive online documentation is available [20], where detailed explanations on all the CPACS nodes can be found.

\subsection{RCE: Process Integration and Execution Framework}

Setting up and running a distributed collaborative design process can produce any number of technical and administrative obstacles. Challenges range from partners being unable (or unwilling) to distribute their tools, problems during file exchange or mixed up versions to properly executing tools in the correct sequence or simply trying to keep track of the state of the process and the flow of data. The DLRdeveloped open source tool RCE (Remote Component Environment) [3] succeeds at alleviating many of these issues.

Fundamentally, RCE can be operated in server or client mode. The server mode is used to host analysis and design tools and services provided by a specific partner and running locally on the same machine as RCE and make them available to other partners. The tools are integrated in RCE in a way, where they accept clearly specified inputs and provide just as clearly specified outputs. Anything in between, including potentially valuable business logic, remains safely on the machine and is not shared with other participants in the process. Instead, only a simple CLI command must be provided. Tools need to be able to run without user interaction, and ideally should accept a CPACS file as input and provide an updated CPACS file as output.

In client mode, tools provided by servers on the network are listed and can be assembled into workflows. In an intuitive

illustrated by figure 2 . process of moving a CPA involving many partners are visited and called, 1 allow the implementation
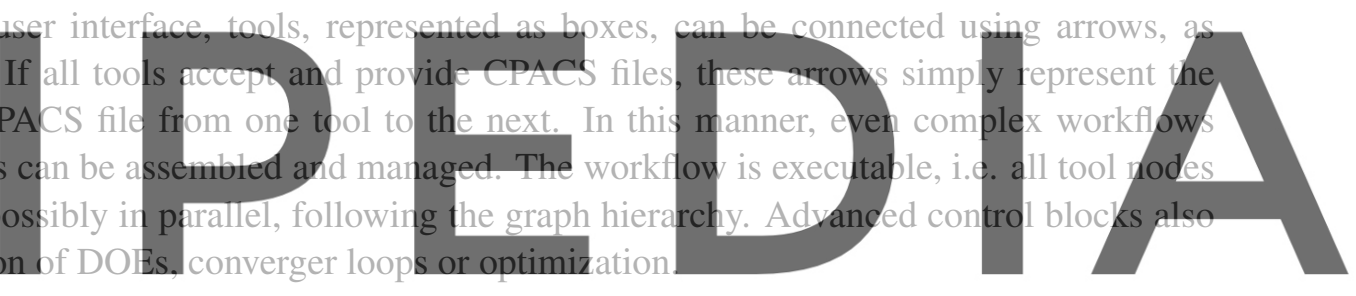

Register for free at https//www.scipedia.com to download the version without the watermark
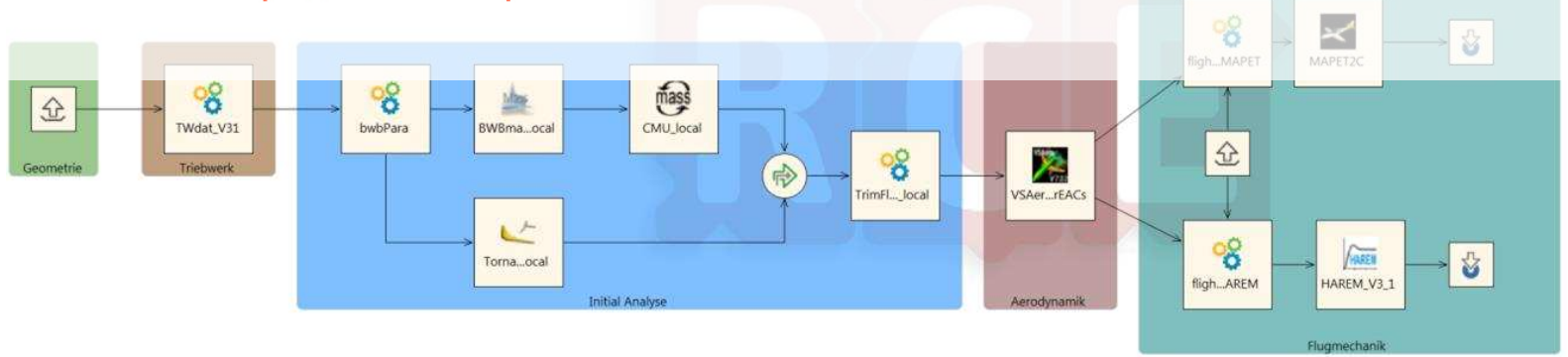

Figure 2: Example of a simple design workflow in RCE (from [16])

\subsection{Design Process and System Architecture}

As more disciplines are taken into consideration, the process of setting up and debugging the design workflow is growing into a task of its own to be performed by a dedicated expert called the integrator. 
Decisions made by the integrator e. g. on the connection of disciplines or the introduction of nested converger loops can have significant effects on the performance and the quality of the results of a workflow. The outcome of the integrator's work is a design process architecture.

However, as the number of disciplinary capabilities continues to increase, the process of manually setting up interdisciplinary design workflows becomes overwhelming. Consequently, several attempts have been made to automate this process by tracing dependencies between tools using graph-based algorithms, taking inspiration from model-based system engineering methodology [23, 24]. This not only facilitates the task of integration, but also allows for fast reconfiguration of the workflow as requirements change and the tool landscape evolves. Rapid deployment of specifically tailored design processes is a key enabler for exploring fundamentally different system architectures, e. g. aircraft configurations, in a limited time frame.

\subsection{Disciplinary Tools - And Experts}

Despite the amount of attention paid to the integration aspects in the above paragraphs, it is indisputable that disciplinary analysis and design capabilities remain the heart and essence of any collaborative design system. By using the term capabilities as opposed to tools, it is implied, that the tool itself is of little use to the integrator, without the insight and judgment of the disciplinary experts. That is, only the disciplinary expert knows how their tool should be used and how the results must be interpreted.

Still, in order to assure compatibility with the integration framework, a few additional requirements for disciplinary tools must be fulfilled, which can be deduced from the above paragraphs.
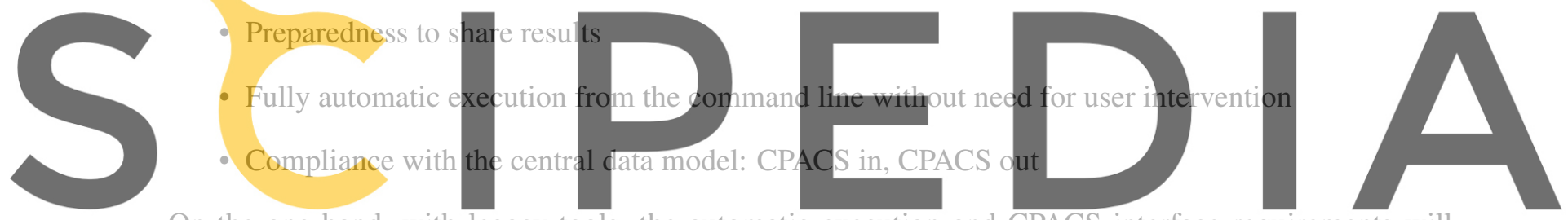

On the one hand, with legacy tools, the automatic execution and CPACS interface requirements will

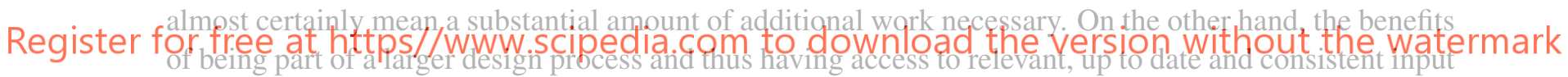
data such as geometry or loads might well be worth the effort.

In the following chapter, application cases from projects where the above technologies have been applied are provided to showcase in more detail what being a disciplinary analysis provider in a collaborative setting involves.

\section{APPLICATION CASES: STRUCTURAL ANALYSIS IN COLLABORATIVE DESIGN PROJECTS}

In the following subsections, disciplinary contributions to three different collaborative design projects are examined using structural analysis in various contexts as an example. First, the potential of fully automatic cross-organizational design workflows is showcased by the AGILE project. Next, the PARSIFAL project illustrates how relevant design information can be assembled to build a workable CPACS data set. Finally, the InDiCaD project provides an perspective beyond classical mechanical analysis by connecting structural layout and cabin design. 


\subsection{AGILE: The Disciplinary Specialist within a Collaborative Design Environment}

The primary goal of the AGILE project [4], was to demonstrate the acceleration of the setup phase for collaborative trans-organizational multidisciplinary design analysis and optimization (MDAO) systems using the AGILE paradigm [25], which takes many of its key cues from the approach outlined above. The methodology was applied in a set of different design use cases, each of which with a different focus.

While rather low fidelity methods were employed for very unconventional configurations in most of the use cases, a consortium consisting of Airbus Defence and Space, CFS Engineering and the DLR, among others, worked on applying higher fidelity methods, such as structural sizing optimization on global FEM level and Euler aerodynamics on a comparatively common unmanned aerial vehicle configuration $[26,5]$. The key challenge in this use case was to integrate the high fidelity analysis capabilities provided by the partners in an automatic and tightly coupled aeroelastic design workflow using RCE. Structural modeling, analysis and sizing capabilities were provided by Airbus Defence and Space, whereas aerodynamic mesh generation and analysis was performed by CFS engineering, with the DLR taking on the role of the integrator.

Figure 3 shows the structural mesh on the one hand, and the aerodynamic surface mesh on the other. Despite originating from different organizations, consistency between the models is ensured, as both have been generated from the same CPACS input data set, using the Descartes model generator [27] and the Sumo mesh generation tool [28] respectively. This is a prerequisite for the application of meshless mapping techniques for load and displacement transfer [29].
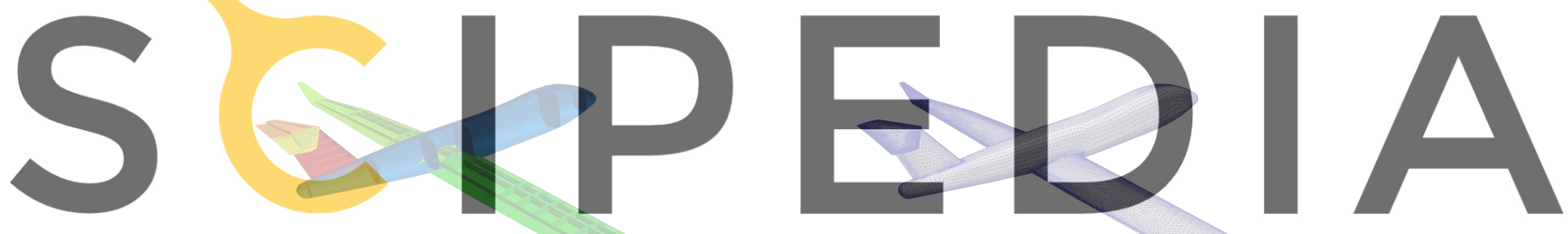

Register for free at https//www.scipedia.com to download the version without the watermark

(a) Structural analysis mesh

(b) Aerodynamic analysis mesh

Figure 3: Comparison of analysis meshes (from [5])

The overall workflow is given in figure 4 as an eXtended Design Structure Matrix (XDSM) [30], a common notation for describing MDAO architectures [31]. The individual disciplines are placed along the main diagonal and linked by off-diagonal entries with vertical and horizontal connections denoting inputs and outputs respectively. Several design loops can be identified, the FSI MDA (fluid-structure interaction multi-disciplinary analysis) loop being the most challenging from an integration perspective. The idea of the loop is to converge the aeroelastic loads and deformations for a given structural design in order to provide a realistic set of loads to a subsequent structural sizing optimization. As illustrated, aerodynamic loads and structural deformations are computed intermittently connected by mapping steps to provide data for the correct points. The entire design process is created and executed in RCE by the integrator at DLR, however the actual disciplinary analyses are performed at different sites. Since the 
data needs to be communicated between different organizational networks, the base features of RCE need to be extended using BRICS technology [32] developed at NLR, which not only allows for seamless data transfer via a neutral server, but also provides protective measures against unwanted data extraction.

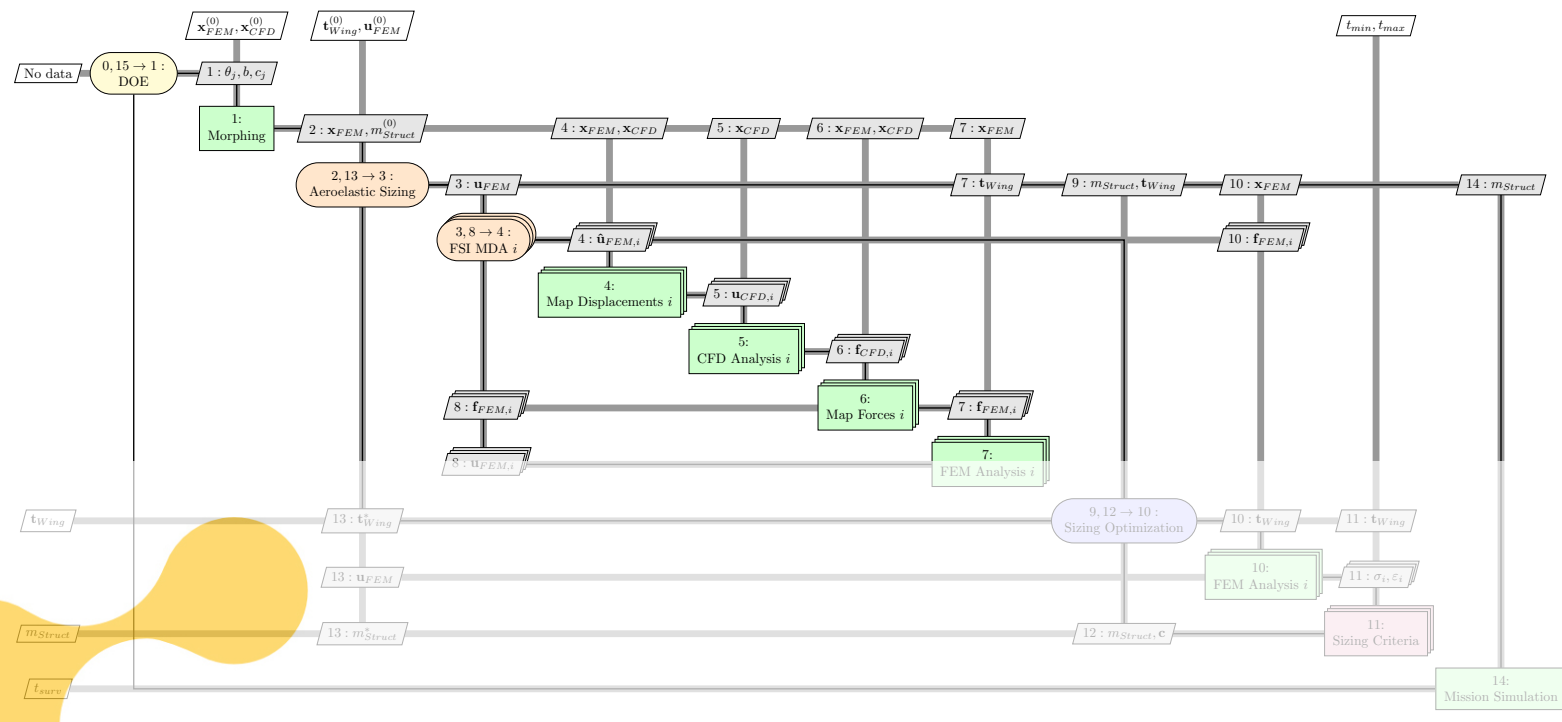

Figure 4: XDSM of the UAV design workflow (from [5])
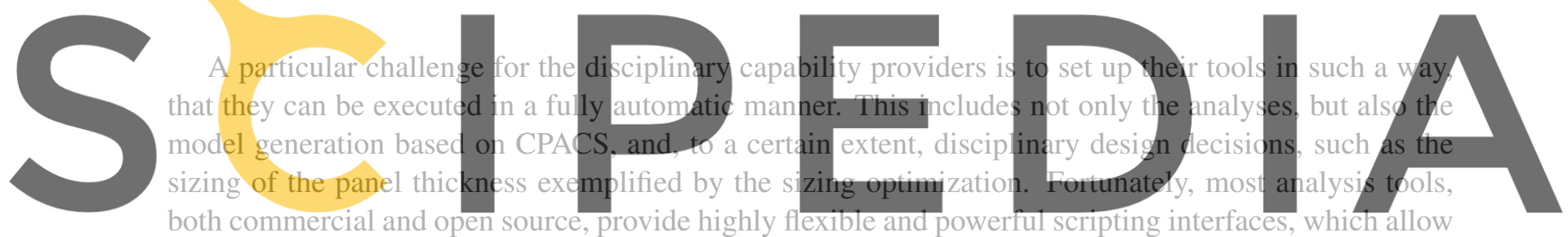

both commercial and open source, provide highly flexible and powerful scripting interfaces, which allow

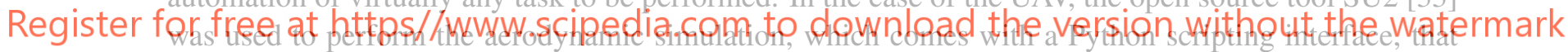

can be used to automate not only the setup and execution of the Euler analysis, but also surrounding tasks

such as the volume mesh deformation. Similariy, the proprietary Lagrange structural soiver [34], which shares many aspect with Nastran and was used to perform the structural analysis and optimization, can also be controlled through Python. In addition, open source packages such as pyNastran [35] further facilitate the modification of analysis decks in order to adapt models or boundary conditions to new inputs provided by the overall process.

Figure 5 illustrates the convergence of the FSI MDA loop, proving that the above process works. It is a testament, not only to the power of integration tools such as RCE, but also to the value of a common product data format like CPACS.

\subsection{PARSIFAL: Assembling Data and Building a Data Set}

Collaborative design is particularly effective, if an existing model is incrementally updated with expert contributions. Usually this model is the outcome of a design synthesis performed using an overall aircraft tool such as openAD [36], the solution of choice in most DLR design projects. At this stage handbook methods or very basic physics simulations are employed to determine a feasible starting point for higher 


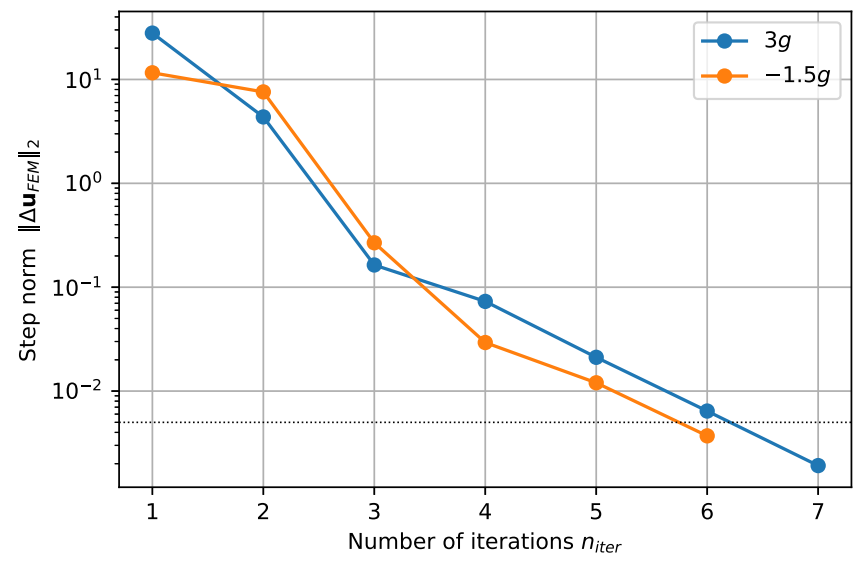

Figure 5: MDA convergence for critical load cases (from [5])

\section{fidelity design activities.}

However, many more exotic layouts tend to exceed the scope of the available traditional synthesizers. This is the case for the boxwing configuration from the PARSIFAL project, which is commonly referred to as Prandtl-Plane. The layout is the outcome of highly specialized design tools, provided by the University of Pisa [37, 1], which do not support CPACS. In order enable distributed design as outlined above, it

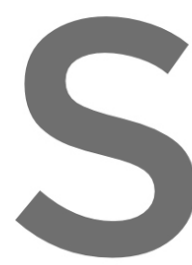

is therefore necessary to

Within the project,

forward and aft wing-fuse

entire configuration.

capabilities provided by
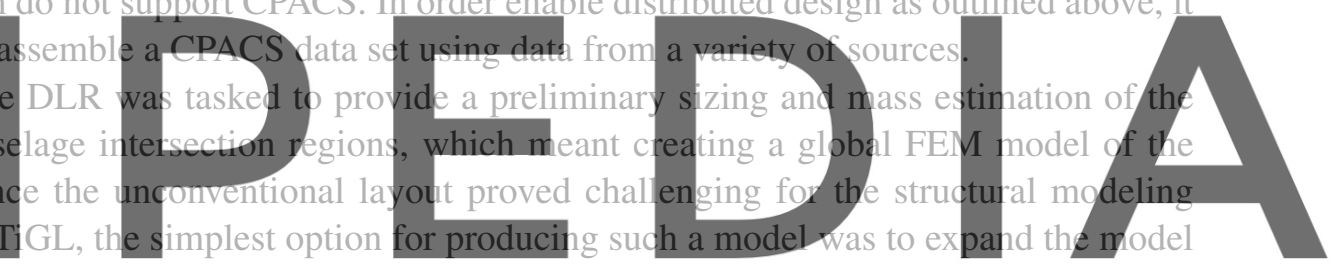

generator by Walther et al. [38], which accepts CPACS as an input. The tool requires a data set containing

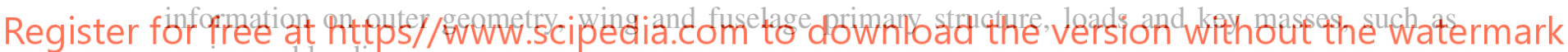
engine and landing gears.

The outer geometry excluding the tail planes was provided by the University of Pisa in IGES format.

Analyzing the model using the Open CASCADE Technology library reveals, that the geometry consists of a collection of NURBS surfaces as illustrated by figure 6a. However, in CPACS the outer loft is defined by a succession of profiles, which are in turn defined by a series of points. In order to compute such profiles, the surfaces from the IGES file must be grouped and concatenated depending on their affiliation to aircraft parts. The resulting merged surfaces are then sampled to yield the necessary profiles for CPACS. Geometry for a tail plane was designed by ONERA [39] and added to the data set during the project, resulting in the complete geometry data set shown in figure $6 \mathrm{~b}$.

Components of the primary structure in CPACS are defined using intersection vectors for the fuselage as described by Scherer and Kohlgrüber [40] and on the wing mid-plane as outlined by Dorbath et al. [41]. The structural information on the Prandtl-Plane was made available by ENSAM in terms of parameters, such as stringer or frame pitch $[42,43]$, which were translated using methods described by Walther and Ciampa [44].

Further input on relevant additional masses such as engines was provided by TU Delft, while the 

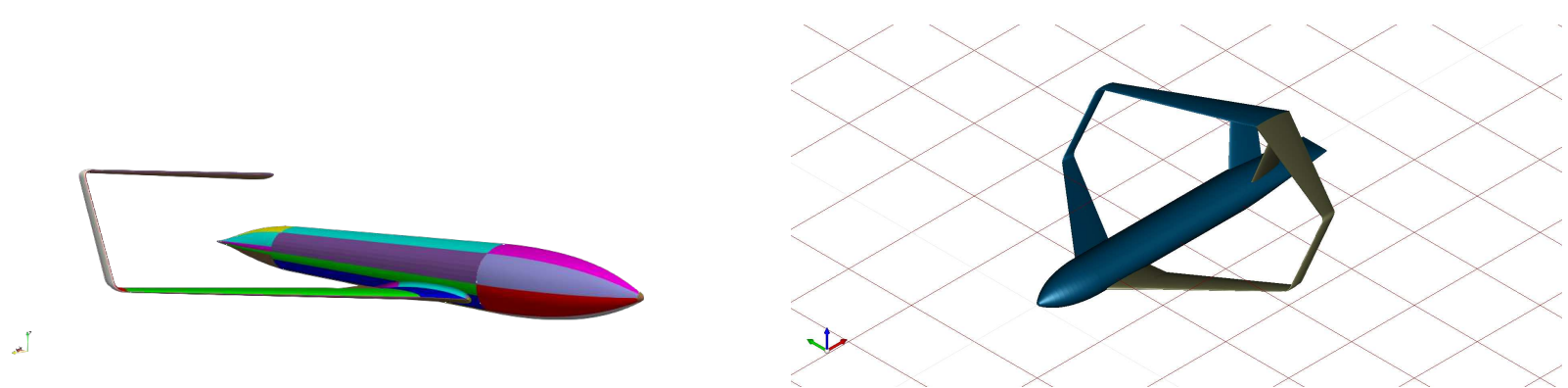

(a) NURBS surface segmentation of the IGES half model by (b) Visualization of the CPACS Prandtl-Plane in TiGL Viewer UNIPI

Figure 6: Conversion of IGES geometry to CPACS

\section{University of Pisa supplied flight loads for sizing maneuver cases, completing the necessary information to go forward with the analysis. Figure 7 shows the resulting component models, which are subsequently joined using multi-point constraint elements to form a valid finite element model of the configuration.}
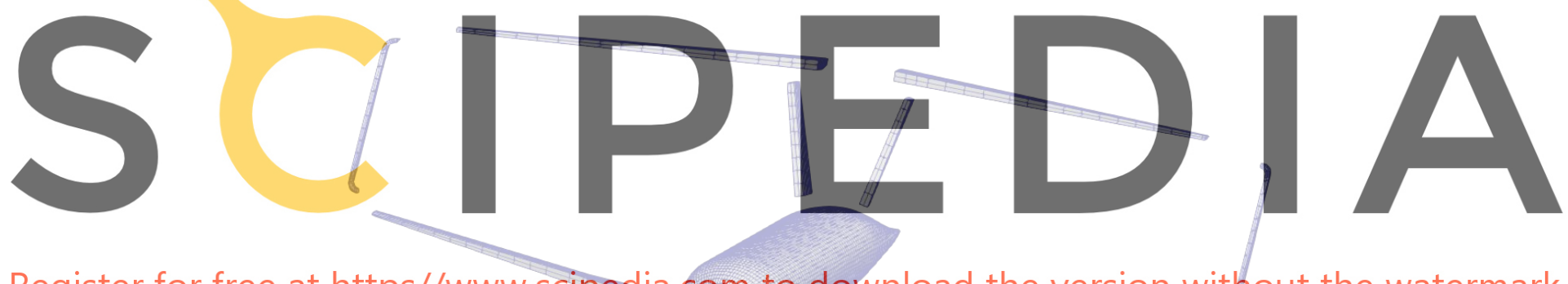

Register for free at https//www.scipedia.com to download the version without the watermark

Figure 7: Component FEM models for the Prandtl-Plane wing system and fuselage

By manipulating the information provided by CPACS, a design of experiments (DOE) was performed on several parameters of the complete model. An example result is given in figure 8 where the outputs of a DOE on the inner and outer skin thickness of the tail plane are plotted. It shows that the tail plane mass can be reduced as the skin thickness is decreased. However, the mass decrease is limited by the critical utilization factor defined as

$$
U F=\frac{\sigma_{r e f}}{S F \cdot \sigma_{c r i t}},
$$

where $\sigma_{r e f}$ is the maximum reference stress present in the elements, $\sigma_{c r i t}$ is the maximum allowable stress for the failure criterion (e. g. von Mises stress for static strength) and $S F$ is a safety factor. Static 
strength and panel buckling after Bruhn [45], which takes into account the panel geometry, are evaluated and the most critical result is selected. For a design to be valid, the condition $U F \leq 1$ must hold. It follows from the plot, that the optimal mass can be achieved if the inner and outer skin thickness are approximately equal.

The manual process of picking an optimum design based on DOE results demonstrated here can also be automated by applying an optimization algorithm. However, as the number of design variables increases, many function evaluations may be necessary, not only for the solutions themselves, but also for the approximation of gradients in the case of gradient based optimizers. As a result, the size of the design space, which can be explored in a given time frame is directly related to the performance of the disciplinary model generation and analysis tools involved.

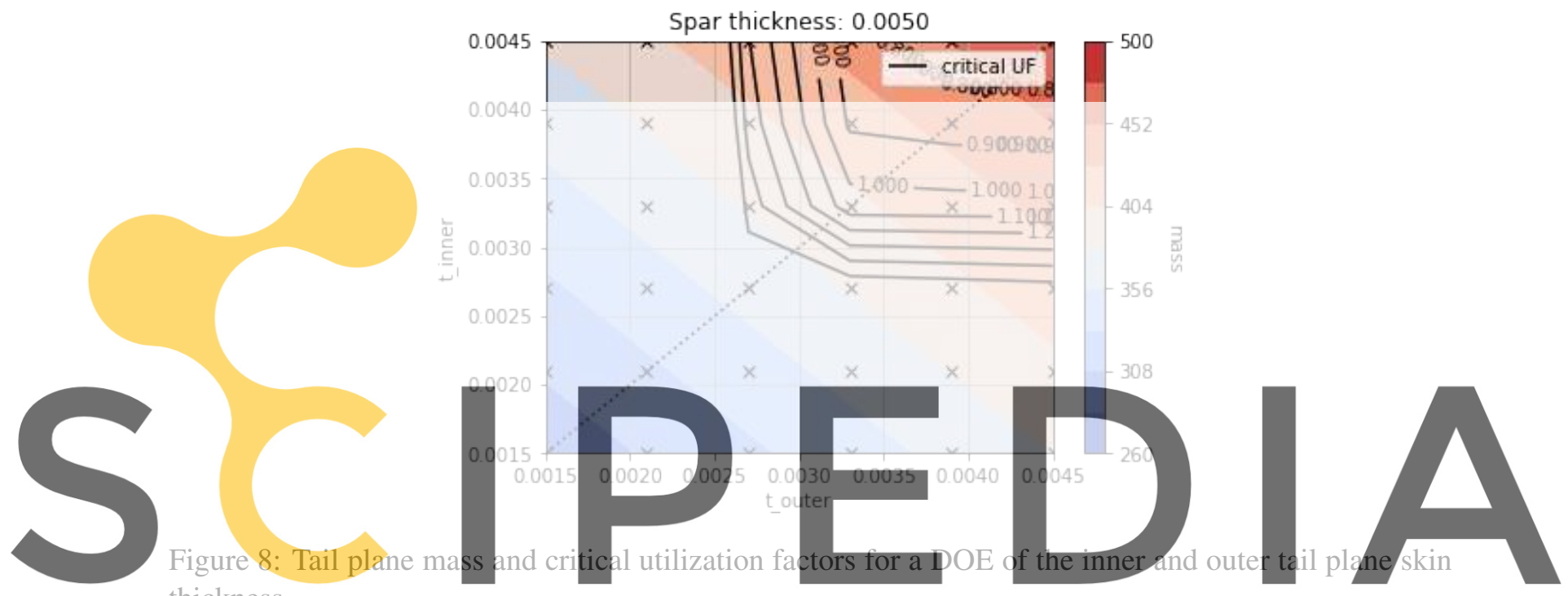
thickness

\section{Register for free at https//www.scipedia.com to download the version without the watermark} Aside from its central role in the above structural modeling and analysis activities, the CPACS data set was used as input for analyses by several other partners, including disciplines such as engines and controls. In fact, by the end of the project, the project partners were able to demonstrate the digital continuity of their tool chain by setting up an automated demo-workflow using RCE.

\subsection{InDiCaD: Incorporating new Design Capabilities}

In order to be able to provide a more complete view of an aircraft design, expanding the pool of available design capabilities is imperative. By expanding on the data provided e. g. by the structural layout generation, new tools can increase the scope and fidelity of a design, while maintaining links to and consistency with the existing tool landscape. Following this approach, the DLR project InDiCaD aims to integrate cabin design and passenger experience aspects to the digital thread, bridging the gap from preliminary design all the way to detailed, interactive virtual reality (VR) environments.

The cabin and structural layout of an aircraft fuselage are closely linked, which means that ideally both should be designed simultaneously. Fuchte [46] did groundbreaking work in cabin design using CPACS, that resulted in the inclusion of a cabin ontology in the schema. As part of the InDiCaD project, 
several expansions of this ontology are being implemented.

Firstly, capacities for referencing detailed component geometry models e. g. of seats, monuments or paneling are added to facilitate the derivation of detailed models for highly immersive VR applications.

Secondly, much effort has been spent on the concurrent and consistent design of cabin and fuselage structure using knowledge-based techniques [8]. To this end, a tool for generating highly detailed CAD geometry models from CPACS as shown in figure 9 has been implemented. This detailed knowledge of the geometry is applied during cabin layout generation e. g. to determine the available 3D space inside the fuselage or to position additional lining panels. Additionally, information on the structural mounts of components can be added, which is useful when building high-fidelity analysis models e. $\mathrm{g}$. for vibro-acoustics.

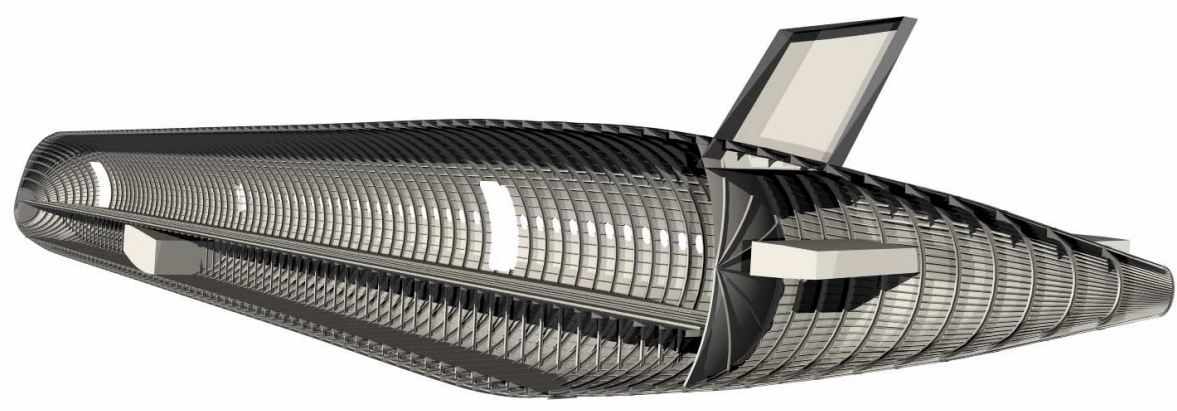

Figure 9: CAD model of a fuselage structure built from CPACS

Another benefit of having CAD geometry available is, that it can easily be triangulated and exported to a VR interface. Combined with a cabin model, an immersive environment can be created, using a VR engine such as Unity [47], where the design can be explored interactively from a passenger's perspective, as shown in figure 10.

\section{CONCLUSION}

In this paper, an integrated collaborative approach to aircraft design has been proposed, to help face future challenges in aviation. Some key enabling technologies have been discussed: A common data format, such as CPACS for unambiguous data exchange, an integration framework like RCE, which enables the assembly and distributed execution of process chains, and system architecting capabilities to manage the increasing complexity of integrated design systems. At the heart of integrated design, however, disciplinary analysis and design capabilities are essential, that combine software and expert knowledge.

Using several collaborative design projects as examples, both the challenges and the benefits for disciplinary specialists have been illustrated. In order to be able to participate in and contribute to an integrated collaborative design process, several requirements must be fulfilled, which might imply that 


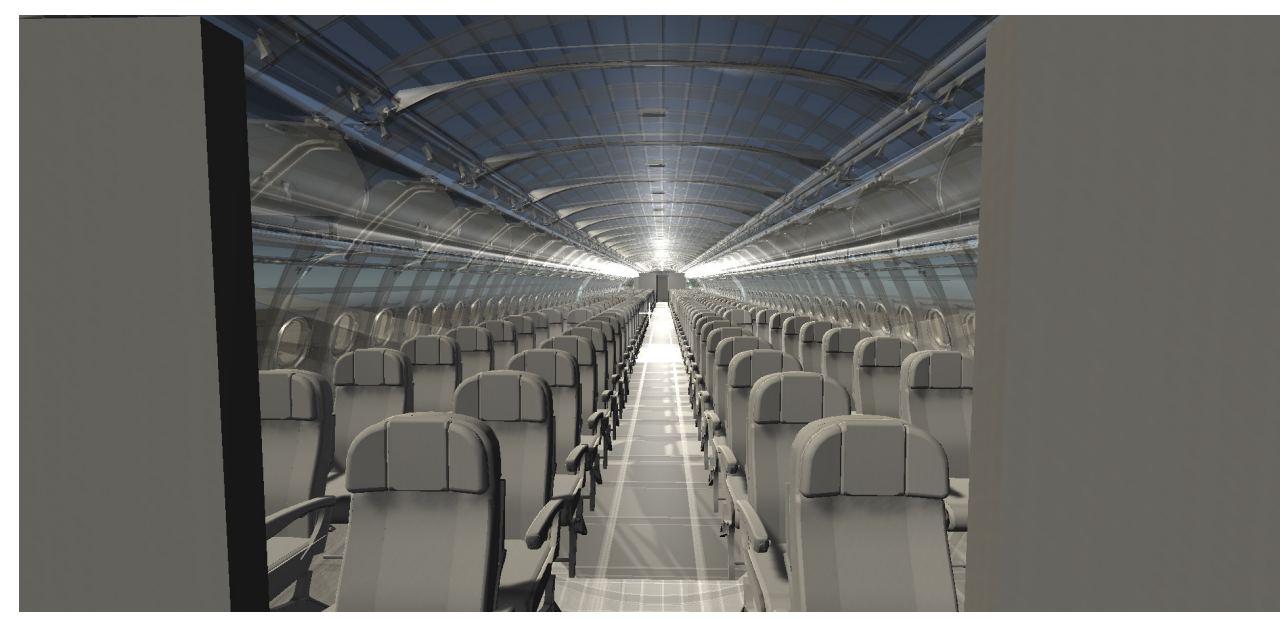

Figure 10: Virtual reality visualization of a single-aisle cabin and underlying structure

a substantial effort must be made up front. First off, the disciplinary analysis tool must be built to run automatically without need for user intervention. While many analysis tools provide powerful scripting frameworks, this might necessitate large amounts of programming. In addition, tools must provide interfaces to the exchange format, for both generating disciplinary models and communicating design decisions, which might not match existing legacy formats. Beyond the purely technical scope, company IT policy or intellectual property concerns might pose obstacles to open data exchange, which can, however, often be alleviated as shown in the AGILE project.

On the other hand, being able to access data provided by partners may lead to better and more relevant analysis results, which can easily be traced back to an overall aircraft design. It also gives an understanding of the interdisciplinary dependencies, which can have a very positive effect on the disciplinary analysis. Automating and parametrizing tools in a structured manner can help make them more robust and flexible, as more and more designs are being investigated, and supporting libraries such as TiGL are a great help along the way. As new requests are made by partners, the scope of one's analysis capability grows naturally over time. Finally, finding new ways to work with existing data and adding new design capabilities might open up entirely new fields where information provided by the disciplinary analysis tool is beneficial.

Ultimately, the quality of the results of collaborative designs will depend on the people who perform the analyses. As such, integrators will always need to rely on a community of experts to adopt certain technologies and share their expertise. With CPACS, TiGL and RCE, the DLR has made a proposal, how some of the key issues in integration can be overcome. However, these solutions are not final, but a work in progress, resulting from years of exchange and discussions between integrators, developers and disciplinary experts. All the tools have been published as open source software via GitHub [48, 49], where users can join the discussion and make suggestions or even actively participate in the development. In this way, hopefully, many more people can be convinced to adopt these technologies and help design the future of aviation - collaboratively. 


\section{REFERENCES}

[1] K. Abu Salem, V. Binante, V. Cipolla, and M. Maganzi. "PARSIFAL Project: a Breakthrough Innovation in Air Transport." In: Aerotecnica Missili \& Spazio 97.1 (Jan. 2018), pp. 40-46. DOI: 10.1007/BF03404764.

[2] Marko Alder, Erwin Moerland, Jonas Jepsen, and Björn Nagel. "Recent Advances in Establishing a Common Language For Aircraft Design With CPACS." In: Aerospace Europe Conference AEC2020, Bordeaux, France, 2020. Jan. 2020.

[3] Doreen Seider, Philipp M. Fischer, Markus Litz, Andreas Schreiber, and Andreas Gerndt. "Open Source Software Framework for Applications in Aeronautics and Space.” In: IEEE Aerospace Conference, Big Sky, Montana, USA. Mar. 2012.

[4] Pier Davide Ciampa et al. "Streamlining Cross-Organizational Aircraft Development: Results from the AGILE Project." In: AIAA Aviation 2019 Forum. American Institute of Aeronautics and Astronautics, June 2019. DOI: 10. 2514/6.2019-3454.

[5] J.-N. Walther, A.-A. Gastaldi, R. Maierl, A. Jungo, and M. Zhang. "Integration aspects of the collaborative aero-structural design of an unmanned aerial vehicle." In: CEAS Aeronautical Journal 11.1 (Aug. 2019), pp. 217-227. DOI: 10.1007/s13272-019-00412-2.

[6] Vittorio Cipolla, Aldo Frediani, Karim Abu Salem, Marco Picchi Scardaoni, and Vincenzo Binante. "The Project "Parsifal": Prandtlplane Architecture For The Sustainable Improvement Of Future Airplanes." In: MATEC Web of Conferences 304 (2019). Ed. by S. Pantelakis and C. Charitidis, p. 01024. DOI: 10.1051/matecconf /201930401024.

[7] F. Reimer et al. "Applikation des Design Thinking Ansatzes auf den Flugzeugkabinen Entwurfsprozess.” de. In: Deutsche Gesellschaft für Luft- und Raumfahrt - Lilienthal-Oberth e.V., 2020. DOI: $10.25967 / 530203$.

[8] Jan-Niclas Walther, Bahadir Kocacan, Christian Hesse, Alex Gindorf, and Björn Nagel. "Automatisierte Kabinenvirtualisierung auf Basis von Flugzeugvorentwurfsdaten.” In: Deutscher Luft- und Raumfahrtkongress 2020. Sept. 2020.

[9] Bundesministerium für Wirtschaft und Energie (BMWi). Die Luftfahrtstrategie der Bundesregierung. 2014.

[10] Helmholtz-Gemeinschaft. Das Programm Luftfahrt. Aug. 2020. URL: https : / /www . helmholtz . de/forschung/luftfahrt_raumfahrt_und_verkehr/luftfahrt/.

[11] Deutsches Zentrum für Luft- und Raumfahrt e.V. (DLR). Programm und Strategie: Luftfahrtforschung im DLR. Aug. 2020. URL: https://www.dlr.de/content/de/artikel/luftfahrt/ luftfahrtforschung/programm-und-strategie-luftfahrtforschung-im-dlr.html.

[12] Carsten M. Liersch and Martin Hepperle. "A distributed toolbox for multidisciplinary preliminary aircraft design.” In: CEAS Aeronautical Journal 2.1-4 (Aug. 2011), pp. 57-68. DOI: 10 . 1007 / s13272-011-0024-6.

[13] Arne Bachmann, Markus Kunde, Markus Litz, and Andreas Schreiber. "A Dynamic Data Integration Approach to Build Scientific Workflow Systems." In: (May 2009). DOI: 10 .1109/GPC . 2009.15.

[14] N. Kroll et al. "DLR project Digital-X: towards virtual aircraft design and flight testing based on high-fidelity methods.” In: CEAS Aeronautical Journal 7.1 (Dec. 2015), pp. 3-27. DOI: 10.1007/ s13272-015-0179-7. 
[15] Erwin Moerland, Sebastian Deinert, Fernaß Daoud, Jochen Dornwald, and Björn Nagel. "Collaborative aircraft design using an integrated and distributed multidisciplinary product development process." In: ICAS 2016. Sept. 2016. URL: https ://elib.dlr.de/111005/.

[16] T. Pfeiffer et al. "Ergebnisse des Flugzeugvorentwurfprojekts "FrEACs" (Future Enhanced Aircraft Configurations).” In: Deutscher Luft-und Raumfahrtkongress. 2017.

[17] Stefan Görtz, Andreas Krumbein, Markus Ritter, and Johannes Hofmann. "DLR-Projekt VicToria - Virtual Aircraft Technology Integration Platform.” In: Deutscher Luft- und Raumfahrtkongress 2018. 2018. URL: https://elib.dlr.de/121695/.

[18] Sebastian Wöhler, Johannes Hartmann, Eric Prenzel, and Harry Kwik. "Preliminary aircraft design for a midrange reference aircraft taking advanced technologies into account as part of the AVACON project for an entry into service in 2028." In: Deutscher Luft- und Raumfahrtkongress 2018. Aug. 2018. URL: https://elib.dlr.de/126000/.

[19] B. Nagel et al. "Communication in Aircraft Design: Can We Establish a Common Language?" In: 28th Congress of the International Council of the Aeronautical Sciences (ICAS), Brisbane, Australia. 2012.

[20] DLR Institute for System Architectures in Aeronautics. CPACS Website. Aug. 2018. URL: http: //cpacs.de.

[21] Martin Siggel, Jan Kleinert, Tobias Stollenwerk, and Reinhold Maierl. "TiGL: An Open Source Computational Geometry Library for Parametric Aircraft Design.” In: Mathematics in Computer Science 13.3 (July 2019), pp. 367-389. DOI: 10.1007/s11786-019-00401-y.

[22] Open Cascade SAS. Open Cascade Website. 2020. URL: www .opencascade .com.

[23] I. van Gent. "Agile MDAO Systems: A Graph-based Methodology to Enhance Collaborative Multidisciplinary Design." PhD thesis. 2019. DOI: 10 . 4233/uuid : c42b30ba-2ba7-4fff - bf 1cf81f85e890af.

[24] Andreas Page Risueño, Jasper Bussemaker, Pier Davide Ciampa, and Bjoern Nagel. "MDAx: Agile Generation of Collaborative MDAO Workflows for Complex Systems." In: (June 2020). DOI: $10.2514 / 6.2020-3133$.

[25] Pier Davide Ciampa and Björn Nagel. "AGILE Paradigm: The next generation collaborative MDO for the development of aeronautical systems." In: Progress in Aerospace Sciences 119 (Nov. 2020), p. 100643. DOI: $10.1016 / j$. paerosci.2020.100643.

[26] R. Maierl et al. "Aero-structural Optimization of a MALE Configuration in the AGILE MDO Framework." In: 31st Congress of the International Council the Aeronautical Sciences (ICAS), Belo Horizonte, Brazil. Sept. 2018. URL: https ://www . icas .org/ICAS_ARCHIVE/ICAS2018/ data/papers/ICAS2018_0675_paper.pdf.

[27] R. Maierl, Ö. Petersson, F. Daoud, and K. U. Bletzinger. "Automatic Generation of Aeroelastic Simulation Models Combined with a Knowledge Based Mass Prediction." In: 5th CEAS Air \& Space Conference, Delft, The Netherlands. 2015.

[28] Maximilian Tomac. "Towards Automated CFD for Engineering Methods in Aircraft Design." PhD thesis. KTH Royal Institute of Technology, Stockholm, Sweden, 2014.

[29] Armin Beckert and Holger Wendland. "Multivariate interpolation for fluid-structure-interaction problems using radial basis functions.” In: Aerospace Science and Technology 5.2 (2001), pp. 125134. DOI: $10.1016 / \mathrm{s} 1270-9638(00) 01087-7$.

[30] Andrew B. Lambe and Joaquim R. R. A. Martins. "Extensions to the design structure matrix for the description of multidisciplinary design, analysis, and optimization processes." In: Structural 
and Multidisciplinary Optimization 46.2 (Jan. 2012), pp. 273-284. DOI: 10.1007/s00158-0120763-y.

[31] Joaquim R. R. A. Martins and Andrew B. Lambe. "Multidisciplinary Design Optimization: A Survey of Architectures.” In: AIAA Journal 51.9 (Sept. 2013), pp. 2049-2075. DOI: 10.2514/1. J051895.

[32] Erik H. Baalbergen, Willem F. Lammen, and Johan Kos. "Mastering restricted network access in aeronautic collaborative engineering across organizational boundaries." In: PDT Europe 2012, The Hague, The Netherlands. Sept. 2012.

[33] Thomas D. Economon, Francisco Palacios, Sean R. Copeland, Trent W. Lukaczyk, and Juan J. Alonso. "SU2: An Open-Source Suite for Multiphysics Simulation and Design." In: AIAA Journal 54.3 (Mar. 2016), pp. 828-846. DOI: 10.2514/1. j053813.

[34] Gerd Schuhmacher, Fernass Daoud, Ögmundur Petersson, and Markus Wagner. "Multidisciplinary Airframe Design Optimization." In: 28th International Congress of the Aeronautical Sciences, Brisbane. 2012.

[35] Steve Doyle. pyNastran - A Python-based interface tool for Nastran's file formats. Feb. 2021. URL: https://github.com/SteveDoyle2/pyNastran.

[36] Sebastian Wöhler, Georgi Atanasov, Daniel Silberhorn, Benjamin Fröhler, and Thomas Zill. "Preliminary Aircraft Design within a Multidisciplinary and Multifidelity Design Environment." In: Aerospace Europe Conference 2020. Apr. 2020. URL: https://elib.dlr.de/135245/.

[37] Marco Picchi Scardaoni, Vincenzo Binante, and Vittorio Cipolla. "WAGNER: a new code for parametrical structural study of fuselages of civil transport aircraft." In: Aerotecnica Missili \& Spazio 96.3 (2017). ISSN: 03657442. DOI: 10.19249/ams .v96i3.311.

[38] Jan-Niclas Walther, Michael Petsch, and Dieter Kohlgrüber. "Modeling of CPACS-based fuselage structures using Python." In: Aircraft Engineering and Aerospace Technology (2017). DOI: 10 . 1108/AEAT-01-2017-0028.

[39] Marco Carini, Michaël Méheut, and Laurent Sanders. "Aerodynamic and Acoustic analysis of a preliminary PrandtlPlane configuration within the framework of the PARSIFAL project." In: AIDAA 2019, Rome, Italy. Sept. 2019.

[40] Julian Scherer and Dieter Kohlgrüber. "Fuselage structures within the CPACS data format." In: Aircraft Engineering and Aerospace Technology 88.2 (Mar. 2016), pp. 294-302. DOI: 10.1108/ aeat-02-2015-0056.

[41] F Dorbath, B Nagel, and V Gollnick. "Extended physics-based wing mass estimation in early design stages applying automated model generation." In: Proceedings of the Institution of Mechanical Engineers, Part G: Journal of Aerospace Engineering 228.7 (Apr. 2013), pp. 1010-1019. DOI: 10.1177/0954410013482657.

[42] Enrico Panettieri, Marco Montemurro, Daniele Fanteria, and Francesco Coccia. "Multi-scale LeastWeight Design of a Wing-Box Through a Global/Local Modelling Approach." In: Journal of Optimization Theory and Applications 187.3 (June 2020), pp. 776-799. DOI: 10.1007 / s10957020-01693-y.

[43] Michele Iacopo Izzi, Marco Montemurro, Anita Catapano, and Jérôme Pailhès. "A multi-scale two-level optimisation strategy integrating a global/local modelling approach for composite structures." In: Composite Structures 237 (Apr. 2020), p. 111908. DOI: 10 . 1016/ j . compstruct . 2020.111908. 
[44] J.-N. Walther and P.D. Ciampa. "Knowledge-based automatic Airframe Design using CPACS.” In: Transportation Research Procedia 29 (2018), pp. 427-439. DOI: 10 . 1016/j . trpro . 2018. 02 . 038.

[45] E. F. Bruhn. Analysis and Design of Flight Vehicle Structures. Jacobs Pub, 1973. ISBN: 0961523409.

[46] Jörg Clemens Fuchte. Enhancement of Aircraft Cabin Design Guidelines with Special Consideration of Aircraft Turnaround and Short Range Operations. Tech. rep. Technische Universität Hamburg-Harburg, Apr. 2014. URL: https://elib.dlr.de/89599/.

[47] Unity Technologies. Unity Real-Time Development Platform I 3D, 2D VR \& AR Engine. retrieved 12.07.2020. 2020. URL: https://unity.com/.

[48] DLR Institute for System Architectures in Aeronautics. DLR-SL - GitHub. Feb. 2021. URL: https : //github.com/DLR-SL.

[49] DLR Institute for Software Technology. DLR Institute for Software Technology - GitHub. Feb. 2021. URL: https://github.com/DLR-SC. 